\title{
Nitrogen depletion in the inner Solar System planets linked to the rates of protoplanetary accretion and differentiation
}

\author{
RAJDEEP DASGUPTA ${ }^{1}$, DAMANVEER GREWAL ${ }^{1}$, \\ ALEXANDRA FARNELL ${ }^{2}$ AND TAYLOR HOUGH ${ }^{1}$ \\ ${ }^{1}$ Rice University \\ ${ }^{2}$ Dartmouth College \\ Presenting Author: rajdeep.dasgupta@rice.edu
}

The cause of extreme depletion of life-essential volatiles like nitrogen $(\mathrm{N})$ in the bulk silicate reservoirs of inner Solar System rocky bodies - whether by nebular and/or parent body processes - is not well understood. The presence of $\mathrm{N}$ in non-labile phases of inner Solar System meteorites suggests their abundant availability in the inner disk during the growth of the earliest formed protoplanets. Therefore, syn- or post-accretion processes such as segregation into the core and atmospheric loss to space must have played an important role in the removal of $\mathrm{N}$ from the silicate reservoirs of differentiated precursors. However, the role of protoplanetary differentiation on the fate of major volatiles such as $\mathrm{N}$ and its effect on the dynamics of planetary growth is unknown. Because $\mathrm{N}$ dissolution in magma oceans is dependent on partial pressure of $\mathrm{N}$ and oxygen fugacity, it is an ideal proxy to track volatile re-distribution in protoplanetary bodies as a function of their sizes and growth zones. Using experiments at 3 $\mathrm{GPa} / 1600-1800{ }^{\circ} \mathrm{C}$ at graphite-absent conditions over a wide $f \mathrm{O}_{2}$ range (IW-7 to IW-1.5), here we show that the siderophile character of $\mathrm{N}$ is an order of magnitude higher than previous estimates [e.g., 1, 2]. The new experimental data combined with coupled metal-silicate-atmosphere fractionation models suggest that asteroid-sized bodies and planetary embryos that grew from them, were N-depleted. However, bodies that became planetary embryo-sized before undergoing large-scale differentiation, had $\mathrm{N}$-rich cores with N-poor silicate reservoirs. Nitrogen in the bulk silicate reservoirs of large Earth-like planets could have been delivered via the cores of the latter type of planetary embryos. Therefore, to satisfy their volatile budgets, the timescales of planetary embryo accretion had to be shorter than their differentiation timescales, i.e., Moon- to Mars-sized planetary embryos grew rapidly within the timescales of ${ }^{26} \mathrm{Al}$ decay. Our study calls for establishing the $\mathrm{N}$ budget of the BSE chiefly via differentiated planetary embryos and obviates the need of late accretion of undifferentiated chondritic materials as the mode of $\mathrm{N}$ delivery to Earth.

[1] Dalou et al. (2017), EPSL 458, 141-151. [2] Grewal et al. (2019), GCA 251, 87-115. 